\title{
1st Workshop on the History of Expressive Systems
}

\author{
James Ryan ${ }^{1}$ and Mark J. Nelson ${ }^{2}$ \\ ${ }^{1}$ Expressive Intelligence Studio, University of California, Santa Cruz \\ jor@soe.ucsc.edu \\ 2 The MetaMakers Institute, Falmouth University \\ mjn@anadrome.org
}

\section{Overview: Topic and Goals}

The first meeting of a new workshop on the History of Expressive Systems (HEX) is being held at ICIDS 2017. By 'expressive systems', we broadly mean computer systems (or predigital procedural methods) that were developed with expressive or creative aims; this is meant to be a superset of the areas called creative AI, expressive AI, videogame AI, computational creativity, interactive storytelling, computational narrative, procedural music, computer poetry, generative art, and more. While much of this purview intersects with projects in artificial intelligence, we are more broadly interested in procedural methods of all kinds (even predigital ones, as mentioned above).

HEX is meant to illuminate and celebrate the history of systems in this area, especially the untold histories of projects that are today forgotten or relatively unknown. Most historical overviews of expressive systems in the literature today are confined to short blurbs in related-works sections, and upon further investigation these brief histories are often at best incomplete. For example, most accounts of the history of story generation cite Sheldon Klein's automatic novel writer [2] as the earliest known system $(e . g .,[1,6])$, but in a recent paper undertaking the HEX initiative we have demonstrated that three other forgotten systems preceded Klein's [3].

Why should we care about old, forgotten work? If we view expressive systems as a vast design space, we can think of each implemented system as an exploratory vessel that ventures into a previously uncharted sector. If these exploratory missions are successful, they signal directions that future systems may move further into to find greater success. When success is not had, the failed projects tell us which areas to avoid. In this way, we learn about spaces that incrementalist research may push further into, dead sectors that we should not return to, and all the other still uncharted areas that we do not know much about at all. Thus, both good and bad systems generate new knowledge that is useful to contemporary and future practitioners. But when we forget about past systems - novel explorations in design space - we lose the knowledge that was generated by those systems: we forget what has been explored and what has not, and which areas are worth exploring further. In our own historical re- 
search, we have discovered systems whose forgotten methods were considered novel when later systems unknowingly reimplemented them decades later.

Beyond these fundamental practical reasons lies the more simple goal of recording an accurate historical record, which encompasses not just a series of names and dates, or a series of system architectures, but also intellectual through lines that trace our fields' histories. Expressive systems are often developed in applied technical areas, but all human endeavor, especially in the area of research, has intellectual underpinnings and emerges out of intellectual contexts. Even in technical areas, there is a history of ideas that undergirds the evolution of systems over time. Returning to practical concerns, good ideas for systems can lead to bad implementations of them, and so we should track ideas too so that we might have another stab at carrying them out well.

Since historical work on expressive systems is important, as we have argued, and since there is little work in this area being done, as we have indicated, we aim to introduce HEX as not just a workshop, but also a new community to provide a home for the first foundational work on the history of this area. In tandem with the first meeting of this workshop, we will also be launching a new repository for historically important expressive systems. This online resource will compile metadata, papers, media coverage, and code listings for historical expressive systems, with a call to action to contemporary researchers and practitioners to reimplement or rationally reconstruct the systems.

Moving forward, we plan to hold HEX at conferences in a variety of fields, with the particular kinds of expressive systems that are of primary interest corresponding to the purview of the host conference. As such, for the first HEX workshop we will emphasize systems related to interactive storytelling and adjacent areas, including story generation, computational narratology, expressive text generation, and so forth.

\section{Format and Schedule}

The first HEX meeting will be a half-day workshop consisting of talks based on submitted papers or abstracts. One of these talks may be an invited keynote presentation from an author of a historically important expressive system, who will give a personal/oral account of its development and intellectual/institutional context. We expect between 10 and 20 total participants.

\section{$3 \quad$ Expected Outcomes}

We will reiterate the following expected outcomes alluded to above:

- Bring together for the first time a community of researchers and practitioners who are interested in the history of expressive systems. Relatedly, we seek to establish a venue that solicits this kind of work and legitimizes it as a full-fledged research area in our larger field. 
- Identify overlooked systems not currently included in the historical canon. A result of such identification could be an initial set of system entries in the forthcoming HEX repository (mentioned above) and an expansive list of promising research topics that could be presented at a future HEX meeting (or related venues).

- Present draft histories for feedback in advance of later publication (in a special issue of a journal, or in a workshop proceedings published on the forthcoming HEX website).

- Increase familiarity with historically used techniques and their successes/failures, with an eye towards updating implementations or reimplementing them, and using that historical knowledge to inform current designs (or analyses of current designs and/or their provenances).

\section{Call for Papers}

We invite researchers interested in the history of expressive systems to participate in the first Workshop on the History of Expressive Systems (HEX1), to be held at ICIDS 2017 in beautiful Funchal, Madeira, on November 14, 2017. ${ }^{3}$ The purpose of this workshop is to improve the historical understanding of our field, both to ensure an accurate historical record for its own sake, but also to bring the history 'into the present' by understanding lines of research and their implications for current work in this rapidly expanding area.

By 'expressive systems', we broadly mean computer systems (or predigital procedural methods) that were developed with expressive or creative aims; this is meant to be a superset of the areas called creative AI, expressive AI, videogame AI, computational creativity, interactive storytelling, computational narrative, procedural music, computer poetry, generative art, and more.

\subsection{Submission Types}

HEX1 will be accepting submissions of the following kinds:

- Abstracts: Papers up to two pages in length, with unlimited additional space for references and appendices (e.g., supporting visual materials). Accepted abstracts will be invited for oral presentation at the workshop.

- Papers: Papers of any length. Accepted papers will be invited for oral presentation at the workshop. Additionally, accepted papers will be invited for publication in the workshop proceedings, which will be made freely available online.

\section{$4.2 \quad$ Scope}

For this first iteration of the workshop held at ICIDS, we prefer a focus on systems within the usual scope of ICIDS, i.e. historical computational narrative

\footnotetext{
${ }^{3}$ Here, we reproduce our call for papers, as distributed prior to the workshop.
} 
systems, videogame narrative, story generation, expressive natural language generation, text bots, e-literature, story understanding, computational narratology, etc. Additionally, histories of the field itself (or specific eras, approaches, etc.) would be a great fit for HEX.

Here are some examples of potential contributions:

- Portraits of forgotten or relatively unknown expressive systems.

- Histories of specific research labs, such as the Yale AI Project led by Roger Schank in the 1970 s.

- Overviews of the careers of unheralded researchers or practitioners, especially those from groups not well represented in the standard histories of the field.

- Reimplementations of early expressive systems, such as Montfort's reimplementation of Strachey's 1952 love-letter generator. ${ }^{4}$

- Rational reconstructions of expressive systems, such as Skald [5] or Wide Ruled [4].

- Reappraisals of conventionally disregarded systems, such as Wardrip-Fruin's extensive overview of TALE-SPIN's underlying processes [6].

- Reframings of known historical systems as expressive systems, or specifically as narrative systems - for example, mainframe war simulations of the 1950s.

- Discussion of obscure computer games as early examples of interactive storytelling, such as Don Daglow's 1973 Star Trek game that extensively featured character dialogue. ${ }^{5}$

- Bringing history into the present: borrowing old techniques for new settings and architectures.

- Bringing the present into history: applying new techniques to old settings and architectures.

- Many more. Not sure if your project is a good fit? Reach out and ask us!

\section{References}

1. Gervas, P.: Computational approaches to storytelling and creativity. AI Magazine 30(3), 49 (2009)

2. Klein, S., et al.: A program for generating reports on the status and history of stochastically modifiable semantic models of arbitrary universes. University of Wisconsin Tech. Rep. TR142 (1971)

3. Ryan, J.: Grimes' fairy tales: A 1960s story generator. In: Proc. International Conference on Interactive Digital Storytelling (2017)

4. Skorupski, J., Jayapalan, L., Marquez, S., Mateas, M.: Wide Ruled: A friendly interface to author-goal based story generation. In: Proceedings of ICVS 2007. pp. 26-37 (2007)

5. Tearse, B., Mawhorter, P., Mateas, M., Wardrip-Fruin, N.: Skald: Minstrel reconstructed. IEEE Transactions on Computational Intelligence and AI in Games 6(2), 156-165 (2014)

6. Wardrip-Fruin, N.: Expressive Processing: Digital fictions, computer games, and software studies. MIT Press (2009)

\footnotetext{
${ }^{4}$ https://nickm.com/memslam/love_letters.html.

${ }^{5}$ https://en.wikipedia.org/wiki/Star_Trek_(script_game).
} 